\title{
Impact of daratumumab on anti-HLA antibodies level in patients with multiple myeloma
}

Bertrand Arnulf ( $\square$ Bertrand.arnulf@aphp.fr)

University of Paris

Floriane Theves

University of Paris

David Kheav

Hôpital Saint-Louis

Alexis Talbot

Hôpital Saint-Louis

Marie-Noelle Peraldi

University of Paris

Camille Villesuzanne

Hôpital Saint-Louis

Stephanie Harel

Hôpital Saint-Louis

Bruno Royer

Hôpital Saint-Louis

Anne-Claire Lepretre

Établissement Français du Sang

Maryvonnick Carmagnat

Hôpital Saint-Louis

Jean-Luc Taupin

University of Paris

\section{Short Report}

Keywords: anti-HLA antibodies, daratumumab, kidney transplantation, multiple myeloma

Posted Date: April 6th, 2022

DOI: https://doi.org/10.21203/rs.3.rs-1412961/v2

License: (9) This work is licensed under a Creative Commons Attribution 4.0 International License.

Read Full License 


\section{Abstract}

Daratumumab, a humanized anti-CD38 monoclonal antibody, has demonstrated anti-plasma cell activity alone and in combination with other drugs in multiple myeloma (MM) and in light chain amyloidosis $(A L)$. Allogenic anti-Human leukocyte antigen (HLA) antibodies are frequently encountered in patients awaiting kidney transplantation, complicating the access to a compatible transplant and represent a major risk factor of humoral rejection. Durably removing the antibodies or eliminating the producing cells is an objective that is currently out of reach. The objectives of our study were to evaluate the frequency of anti-HLA antibodies in MM and AL patients, and to determine the effect of daratumumab on them.

Eligible patients with diagnosed MM or AL were selected $(n=182)$, among whom 84 had anti HLA antibodies (46\%). Forty-nine patients (45 MM and $4 \mathrm{AL}$ ) were retrospectively included.

Patients were treated with a combination of an alkylating agent, an immunomodulatory imide drug (IMiD), a Proteasome inhibitor, with $(n=22)$ or without $(n=22)$ daratumumab. Five patients received daratumumab alone. Treatment with daratumumab lowered anti-HLA antibodies in 85\% (23/27) of cases including complete loss in $56 \%$ of cases (15/27) compared to $77 \%(17 / 22)$ and $45 \%(10 / 22)$ respectively, $p=0.8$.

This study couldn't find a statistic impact of daratumumab to lower HLA antibody in this cohort. The association with chemotherapy could be an important bias and this hypothesis will be evaluated in ongoing studies enrolling patients undergoing kidney transplantation.

\section{Introduction}

Multiple Myeloma ( $\mathrm{MM})$ is characterized by a proliferation of clonal plasma cells highly expressing the CD38 antigen (1). The treatment of MM is based on front-line treatment for young and healthy patients with combinations of steroids, Immunomodulatory imide drug (IMiD), Proteasome inhibitor (PI), Alkylating agents in stem cell transplantation and Daratumumab (2). Daratumumab is a humanized IgG1k monoclonal antibody targeting CD38 tumoral and non-tumoral plasma cells. The mechanism of action of daratumumab comprises immune-mediated effects, including complement-dependent and antibody-dependent cell-mediated cytotoxic effects, antibody-dependent cellular phagocytosis, and apoptosis by cross-linking. Daratumumab has significantly improved the efficiency of treatment in MM, alone in relapsed MM, and especially in combination with PI or IMiDs in relapsed disease and in front line treatment (3)(4)(5). In addition, daratumumab presents an outstanding safety profile with nonconsiderable side effects. Altogether, this suggests it could potentially be used in other non-tumoral plasma cell-mediated pathologies.

The presence of anti-HLA antibodies prior organ transplantation is associated with a decreased number of donors and a longer waiting time. Moreover, anti-HLA antibodies after kidney transplantation promotes hyperacute rejection, acute and chronic humoral rejection, and early graft loss (6). Kidney survival and overall survival are lower in immunized patients with anti-HLA antibodies and chronic humoral rejection is 
nowadays considered as the leading cause of graft loss (7). The standard desensitization protocols include plasmapheresis associated with high dose polyvalent intravenous immunoglobulins (IVIG)(8). However, in strongly immunized patients refractory to conventional treatments the desensitization protocols prior to or after renal transplantation are not standardized. Other therapies are either poorly effective or evaluated in small non-randomized controlled studies such as antibodies targeting IL6 or its receptor, in order to control antibody production, the synthesis of antibodies by inhibition of $B$ lymphocytes and plasma cells, and complement inhibitors, to regulate antibody main effector function (9),(10),(11),(12). Thus, plasma cells are a logical target of therapy given their critical role in antibody production and high level of CD38 expression. Daratumumab could be an effective drug for plasma cell depletion and desensitization.

To measure the effect of daratumumab on serum anti-HLA antibodies level, we studied two cohorts of patients treated or not with daratumumab for symptomatic MM or AL. The objectives of our study were to determine the frequency of anti-HLA antibodies in MM and AL patients and to evaluate the impact of daratumumab on the evolution of anti-HLA antibody levels.

\section{Patients And Methods}

In this retrospective, single-center study at Saint Louis Hospital, eligible patients with symptomatic MM or AL were tested for anti-HLA antibodies during treatment in two sera samples between June 2014 and October 2019. Mean time between two samples were 176 days [21-1384]. All patients provided informed consent. The inclusion criteria were patients with symptomatic MM or AL requiring treatment according to the recommendation from IMWG (International Myeloma Working Group) (13).

Sixty two patients were involved in a test cohort and the frequency of anti-HLA antibodies was evaluated. The results were validated on a second cohort of 120 patients. The aim was to monitor the level of antiHLA antibodies in patients treated with and without daratumumab. Transfusion history for red blood cells, platelets and plasma was obtained from the EFS (établissement français du sang). Cytogenetic characteristics of patients were performed with FISH technique searching for $17 \mathrm{p}$ deletion, $\mathrm{t}(4 ; 14)$, and $\mathrm{t}(11 ; 14)$ translocations.

Daratumumab was used in front line in the "Cassiopeia" study (NCT02541383) or in relapse at the dose of $16 \mathrm{mg} / \mathrm{kg}$ either in monotherapy or in combination with PI or IMiD. Daratumumab was administered intravenously once per week (days 1, 8, 15 and 22) during cycles 1 and 2, once every 2 weeks (on day 1 ) during cycles 3 to 6 , and once every 4 weeks thereafter. In the second group, patients received a treatment combining either Alkylating agent, IMiD, or PI, without daratumumab.

Research and identification of anti-HLA antibodies were carried out in the immunology laboratory of Saint Louis Hospital in Paris using the Luminex technology and the screening kit for anti-HLA antibodies for class I and for class II (ref LSM12, One Lambda, Canoga Park, CA). Results are expressed as a ratio between the highest MFI (Mean Fluorescence Intensity) of the sample against a negative control provided by the manufacturer. Identification of anti-HLA antibody specificities in the positive sera could not be 
performed for this study. The anti-HLA antibody level is considered as reduced if the ratio decreases by at least $40 \%$ in at least one class. Statistical analyzes were performed using Fisher's exact test with $p<0.05$.

\section{Results}

\section{Characteristics of the patients in the test cohort}

The test cohort included 62 patients (median age 63 years, sex ratio M/F = 1,9). Anti-HLA antibodies were found in $23 \%(14 / 62)$ of the patients. Among the 14 patients with positive anti-HLA antibodies, 9 had a second sample available, and anti-HLA antibodies disappeared in 6 of them after daratumumab treatment (Fig. 1). All patients received treatment including PI and IMiD and half received an alkylating agent (Table 1). Most of the patients in this cohort had a previous history of transfusion (7/9).

Table 1

Characteristics of patients in both cohorts at the time of the first sample

\begin{tabular}{lll} 
& Cohort 1 & Cohort 2 \\
\hline Myeloma (n) & 8 & 37 \\
AL amyloidosis (n) & 1 & 3 \\
\hline Pt with anti Class 1 HLA abs (n) & 5 & 33 \\
Pt with anti Class 2 HLA abs (n) & 7 & 26 \\
Pt with anti Class 1 and 2 HLA abs (n) & 3 & 19 \\
\hline Polyclonal gammaglobuline (mean, g/L) & 5.6 & 4.8 \\
\hline Nb of line of treatment (n, range) & 2 [1-6] & 2 [0-4] \\
No treatment (n) & 0 & 11 \\
Alkylating agent (n) & 5 & 11 \\
Imids (n) & 9 & 22 \\
PI (n) & 9 & 26 \\
ASCT (n) & 7 & 15 \\
\hline Total (n) & 9 & 40
\end{tabular}

Pt: patient, abs: antibodies

\section{Evolution of anti-HLA antibodies in the test cohort}


In this cohort, the median time between the first and the second anti-HLA antibody sample was 290 days [40-1384] and in the daratumumab group, the median time was 251 days. 3 patients received daratumumab as monotherapy, and 5 in combination with $\mathrm{PI}$, IMiD, or Alkylating agent. In patients treated with daratumumab, anti-HLA antibodies disappeared in $75 \%$ of cases $(6 / 8)$. Considering both decrease and loss of anti-HLA antibodies, the rate reached $88 \%(7 / 8)$.

\section{Characteristics of patients in the validation cohort}

The validation cohort included 120 patients. Anti-HLA antibodies were detected in 70 patients (58\%). A second sample was available for 42 patients and 2 patients were excluded because the interval between the two samples was less than 5 days. 21 patients showed positive anti-HLA antibodies on their second sample (Fig. 1).

We further studied 40 patients (37 MM and $3 \mathrm{AL}$ ) who received a median of 2 lines of treatment [0-4]. 11 patients were not yet treated at the time of the first sample. Of the 29 patients under treatment, 26 (90\%) received a $\mathrm{PI}, 22$ (76\%) patients received an IMiD, 11 (40\%) received an alkylating agent, and 15 patients (52\%) underwent high dose chemotherapy followed by an autologous stem cell transplant (Table 1).

Half of the patients received a transfusion (red blood cells, platelets and plasma) (20/40). Among the 24 males in this cohort, 13 (33\%) did not have any prior history of transfusion.

\section{Evolution of anti-HLA antibodies in the validation cohort}

In the validation cohort, the median time between the two serum samples was 92 days [21-779 days] and in the daratumumab group, the median time was 63 days. 19 out of 40 patients received treatment including daratumumab, in monotherapy $(n=2)$, and in combination with a PI, IMiD, or alkylating agent $(n=17) .21$ patients received only treatment a PI, IMiD, or alkylating agent. The rate of anti-HLA antibody loss was similar in patients treated with daratumumab $(47 \%)$ or without $(48 \%)$.

A subgroup of 16 out of $19(84 \%)$ patients treated with daratumumab had complete loss or decreased anti-HLA antibody levels. In the subgroup treated without daratumumab, loss or decrease of anti-HLA antibodies was observed in $81 \%(17 / 21)$.

\section{Evolution of anti-HLA antibodies in the whole cohort}

To analyze the effect of daratumumab on a larger number of patients, the two cohorts were combined. Therefore, we considered an overall cohort of 49 patients, including 27 patients treated with daratumumab and 22 patients without. Among the 25 patients with negative anti-HLA antibody levels, 15 patients $(60 \%)$ received daratumumab versus 10 patients $(40 \%)$ without daratumumab, $p=0.57$. 
$85 \%(23 / 27)$ of patients treated with daratumumab, either reduced or completely lost anti-HLA antibodies, as compared to $77 \%(17 / 22)$ for patients treated without daratumumab (Fig. 2$)(p=0.83)$.

In the 25 patients achieving anti-HLA antibodies level complete loss, the median treatment exposure was shorter in the presence of daratumumab than with other therapies: 112 days [27-516] versus 169 days [31-779], respectively (Fig. 2).

\section{Impact of daratumumab as monotherapy or in combination}

Five patients were treated with daratumumab in monotherapy and 22 patients received daratumumab in combination with PI or IMiD: 2 out of 5 patients had negative anti-HLA antibodies levels versus 13 out of 22 patients respectively $(p=0.6)$. In addition, 4 out of $5(80 \%)$ patients treated with daratumumab alone decreased or lost anti-HLA antibody level as compared to 19 out of $22(86 \%)$ other patients, $p=1.0$. Hence, daratumumab alone or in combination should have a similar impact on the anti-HLA antibody level.

\section{Discussion}

The objectives of our study were to evaluate the rate of anti-HLA antibodies in MM and AL, to evaluate the impact of daratumumab on anti-HLA antibodies in a first test cohort $(n=62)$ and to confirm the results in a validation cohort $(n=120)$.

Anti-HLA antibodies were found in $23 \%$ and $53 \%$ in the test and validation cohort, respectively. In the literature anti-HLA antibodies were found in around $20 \%$ in patients with hematological malignancies (14) (15). Patients with prior organ transplantation, pregnancies, or blood transfusions have an increased likelihood of becoming sensitized by developing anti-HLA antibodies (16). In this study, the major risk factor for anti-HLA antibodies was transfusion (78\% and 50\%, respectively), and the majority of patients were males making the risk factor caused by pregnancy unlikely.

In our study, after conventional therapy with a PI, IMiD, or Alkylating agent plus daratumumab, the frequency of anti-HLA antibodies decreased to $58 \%(23 / 40)$ of patients, versus $42 \%(17 / 40)$ without daratumumab. Anti-HLA antibody disappeared $(n=25)$ in $60 \%(15 / 25)$ with daratumumab, versus $40 \%$ $(10 / 25)$ without. A quantitative study with anti-HLA antibodies specificities could be done therefore to assess more precisely the evolution of these antibodies over time. However, no statistically significant difference was found. This could be explained by the small number of patients and the bias with the association with chemotherapy. The effect of daratumumab alone on anti-HLA antibodies is difficult to assess given the few patients that received daratumumab in monotherapy even if there were no statistical difference between the 2 groups of daratumumab (combination and monotherapy).

In the 25 patients who negativized anti-HLA antibodies with and without daratumumab, the median duration of treatment was shorter in the presence of daratumumab than with the other treatments: 112 
days [27-516] versus 169 days [31-779], respectively. Daratumumab could therefore lead to a quicker clearance of anti-HLA antibodies than other therapies (IP, IMiDs, alkylating agents, steroids) within a median time of 3 months. In the treatment of MM, daratumumab in monotherapy acts faster again tumoral plasma cells with a median time to response of 1 month (3).

In preclinical study on macaques immunized, the use of daratumumab pre-transplant allowed significantly longer kidney graft survival (17). Also in a case report on kidney transplantation, daratumumab helped to decrease anti HLA antibody and improved antibody-mediated rejection (18). A phase 1-2 study (NCT04204980) is underway on the use of daratumumab to desensitize highly immunized patients awaiting kidney transplantation. The first step is a dose escalated step from $4 \mathrm{mg} / \mathrm{kg}$ to $16 \mathrm{mg} / \mathrm{kg}$. For the second step, patients will receive daratumumab at a dose of $16 \mathrm{mg} / \mathrm{kg}$ weekly for 8 weeks.

A remaining open question is the optimal dose and schedule of daratumumab for this particular purpose. In fact, the monoclonal anti-CD20 antibody rituximab is used at different doses in lymphoproliferative syndromes and in dysimmune pathologies (19), (20).

In conclusion, this study couldn't find a significant effect of daratumumab to lower HLA antibody. The association of chemotherapy in both group is an important bias. We find nevertheless a quicker clearance of anti-HLA in daratumumab group. These results need to be confirmed in larger trials including immunized patients waiting for a transplant or with acute humoral rejection.

\section{Declarations}

\section{Availability of data and materials}

The datasets used during the current study are available from the corresponding author on reasonable request.

\section{Acknowledgements}

We would like to thank all the patients and healthcare professionals participating in the study.

\section{Funding}

Not applicable.

\section{Author information}

Affiliations 
Immuno-hematology department, Saint Louis hospital, APHP Université de Paris

Floriane Theves, Alexis Talbot, Camille Villesuzanne, Stephanie Harel, Bruno Royer, Bertrand Arnulf

Nephrology and transplantation department, Saint Louis hospital, APHP Université de Paris

Marie-Noelle Peraldi

Immunology laboratory, Saint Louis hospital, APHP Université de Paris

David Kheav, Maryvonnick Carmagnat, Jean-Luc Taupin

Transfusion Department, Etablissement Français Du Sang, Site Saint-Louis, Paris.

Anne-Claire Lepretre

\section{Authors' Contributions}

B.A,D.K,F.T conceived the original idea and designed the study. F.T, D.K, S.H, C.V, A.T, B.R, B.A, AC.L, JL.T collected the data for the study, which were analyzed by F.T and D.K. The data interpretation and manuscript drafting were performed by F.T, B.A, MN.P, JL.T.

F.T, A.T, MN.P, C.V, S.H, B.R, AC.L, JL.T, M.C, B.A reviewed and edited the manuscript and gave final approval for submission.

\section{Corresponding author}

Correspondence to Bertrand Arnulf

\section{Ethics declarations}

\section{Conflict of interest}

The authors declare that they have no conflict of interest.

\section{Ethical approval}

All patients provided informed consent.

\section{References}


1. Lin P, Owens R, Tricot G, Wilson CS. Flow cytometric immunophenotypic analysis of 306 cases of multiple myeloma. Am J Clin Pathol. avr 2004;121(4):482-8.

2. Rajkumar SV, Kumar S. Multiple Myeloma: Diagnosis and Treatment. Mayo Clin Proc. janv 2016;91(1):101-19.

3. Lokhorst HM, Plesner T, Laubach JP, Nahi H, Gimsing P, Hansson M, et al. Targeting CD38 with Daratumumab Monotherapy in Multiple Myeloma. N Engl J Med. 24 sept 2015;373(13):1207-19.

4. Dimopoulos MA, Oriol A, Nahi H, San-Miguel J, Bahlis NJ, Usmani SZ, et al. Daratumumab, Lenalidomide, and Dexamethasone for Multiple Myeloma. N Engl J Med. 06 2016;375(14):1319-31.

5. Moreau P, Attal M, Hulin C, Arnulf B, Belhadj K, Benboubker L, et al. Bortezomib, thalidomide, and dexamethasone with or without daratumumab before and after autologous stem-cell transplantation for newly diagnosed multiple myeloma (CASSIOPEIA): a randomised, open-label, phase 3 study. The Lancet. 6 juill 2019;394(10192):29-38.

6. Patel R, Terasaki PI. Significance of the Positive Crossmatch Test in Kidney Transplantation. N Engl J Med. 3 avr 1969;280(14):735-9.

7. Montgomery RA, Lonze BE, King KE, Kraus ES, Kucirka LM, Locke JE, et al. Desensitization in HLAincompatible kidney recipients and survival. N Engl J Med. 28 juill 2011;365(4):318-26.

8. Wan SS, Ying TD, Wyburn K, Roberts DM, Wyld M, Chadban SJ. The Treatment of Antibody-Mediated Rejection in Kidney Transplantation: An Updated Systematic Review and Meta-Analysis. Transplantation. avr 2018;102(4):557-68.

9. Eskandary F, Bond G, Schwaiger E, Kikic Z, Winzer C, Wahrmann M, et al. Bortezomib in late antibodymediated kidney transplant rejection (BORTEJECT Study): study protocol for a randomized controlled trial. Trials. 3 avr 2014;15:107.

10. Sautenet B, Blancho G, Büchler M, Morelon E, Toupance O, Barrou B, et al. One-year Results of the Effects of Rituximab on Acute Antibody-Mediated Rejection in Renal Transplantation: RITUX ERAH, a Multicenter Double-blind Randomized Placebo-controlled Trial. Transplantation. févr 2016;100(2):391-9.

11. Montgomery RA, Orandi BJ, Racusen L, Jackson AM, Garonzik-Wang JM, Shah T, et al. PlasmaDerived C1 Esterase Inhibitor for Acute Antibody-Mediated Rejection Following Kidney Transplantation: Results of a Randomized Double-Blind Placebo-Controlled Pilot Study. Am J Transplant Off J Am Soc Transplant Am Soc Transpl Surg. 2016;16(12):3468-78.

12. Choi J, Aubert O, Vo A, Loupy A, Haas M, Puliyanda D, et al. Assessment of Tocilizumab (AntiInterleukin-6 Receptor Monoclonal) as a Potential Treatment for Chronic Antibody-Mediated Rejection and Transplant Glomerulopathy in HLA-Sensitized Renal Allograft Recipients. Am J Transplant Off J Am Soc Transplant Am Soc Transpl Surg. sept 2017;17(9):2381-9.

13. Rajkumar SV, Dimopoulos MA, Palumbo A, Blade J, Merlini G, Mateos M-V, et al. International Myeloma Working Group updated criteria for the diagnosis of multiple myeloma. Lancet Oncol. nov 2014;15(12):e538-548. 
14. Ruggeri A, Rocha V, Masson E, Labopin M, Cunha R, Absi L, et al. Impact of donor-specific anti-HLA antibodies on graft failure and survival after reduced intensity conditioning-unrelated cord blood transplantation: a Eurocord, Société Francophone d'Histocompatibilité et d'Immunogénétique (SFHI) and Société Française de Greffe de Moelle et de Thérapie Cellulaire (SFGM-TC) analysis. Haematologica. juill 2013;98(7):1154-60.

15. Ciurea SO, Thall PF, Wang X, Wang SA, Hu Y, Cano P, et al. Donor-specific anti-HLA Abs and graft failure in matched unrelated donor hematopoietic stem cell transplantation. Blood. 24 nov 2011;118(22):5957-64.

16. Lopes D, Barra T, Malheiro J, Tafulo S, Martins L, Almeida M, et al. Effect of Different Sensitization Events on HLA Alloimmunization in Kidney Transplantation Candidates. Transplant Proc. mai 2015;47(4):894-7.

17. Kwun J, Matignon M, Manook M, Guendouz S, Audard V, Kheav D, et al. Daratumumab in Sensitized Kidney Transplantation: Potentials and Limitations of Experimental and Clinical Use. J Am Soc Nephrol JASN. 2019;30(7):1206-19.

18. Doberer K, Kläger J, Gualdoni GA, Mayer KA, Eskandary F, Farkash EA, et al. CD38 Antibody Daratumumab for the Treatment of Chronic Active Antibody-mediated Kidney Allograft Rejection. Transplantation. 1 févr 2021;105(2):451-7.

19. McLaughlin P, Grillo-López AJ, Link BK, Levy R, Czuczman MS, Williams ME, et al. Rituximab chimeric anti-CD20 monoclonal antibody therapy for relapsed indolent lymphoma: half of patients respond to a four-dose treatment program. J Clin Oncol Off J Am Soc Clin Oncol. août 1998;16(8):2825-33.

20. Edwards JCW, Szczepański L, Szechiński J, Filipowicz-Sosnowska A, Emery P, Close DR, et al. Efficacy of B-Cell-Targeted Therapy with Rituximab in Patients with Rheumatoid Arthritis [Internet]. http://dx.doi.org/10.1056/NEJMoa032534. Massachusetts Medical Society; 2009 [cité 1 mars 2021]. Disponible sur: https://www.nejm.org/doi/10.1056/NEJMoa032534

\section{Figures}

\section{Figure 1}

Flow chart of the test and validation cohorts 


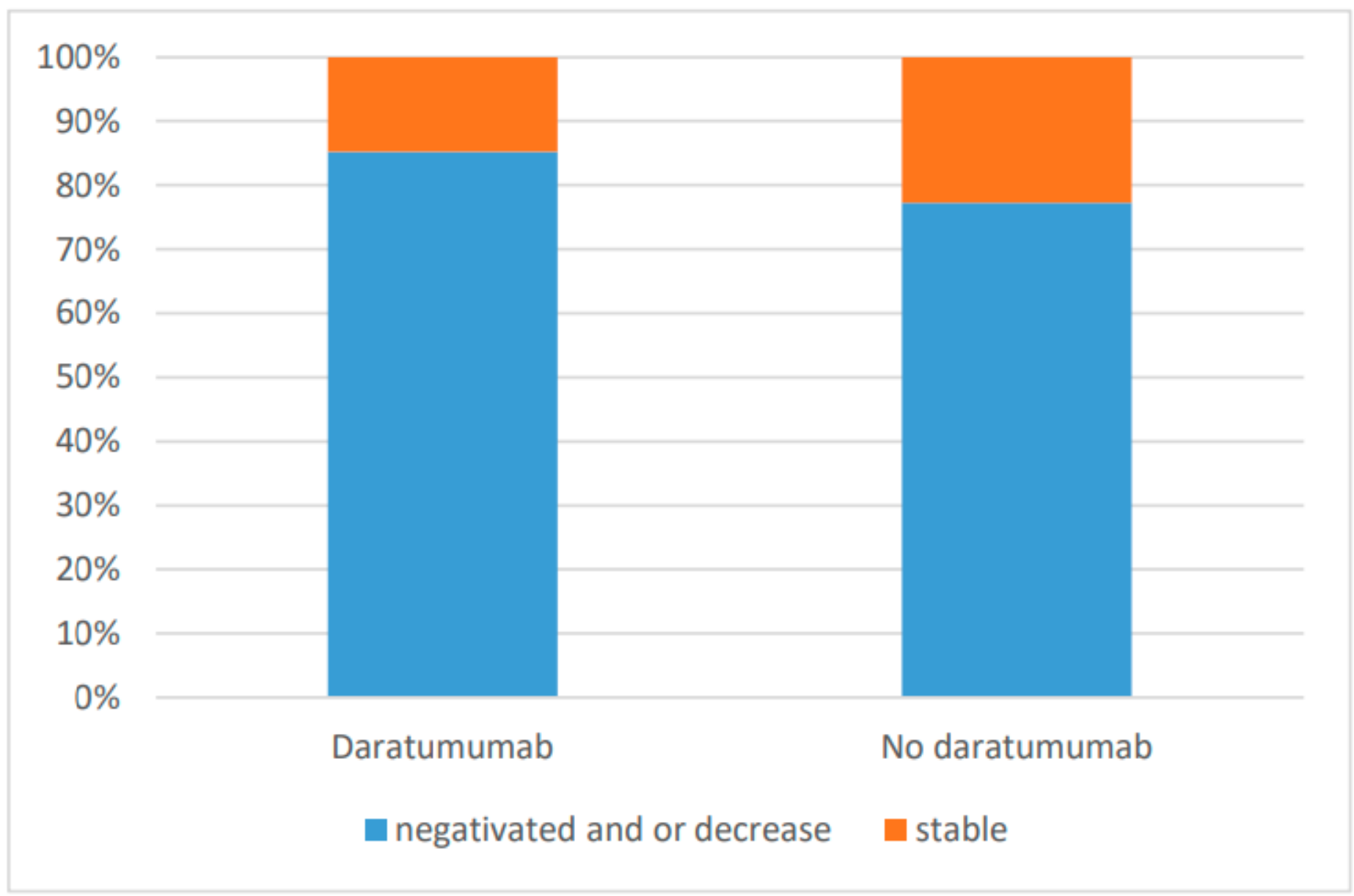

Figure 2

Evolution of HLA antibody according to daratumumab in the global cohort 